Subscriber access provided by Caltech Library

\title{
Article
}

\section{Analysis and control of chain mobility in protein hydrogels}

\author{
Peter B Rapp, Ahmad K. Omar, Jeff J. Shen, Maren E. Buck, Zhen-Gang Wang, and David A. Tirrell
}

J. Am. Chem. Soc., Just Accepted Manuscript • DOI: 10.1021/jacs.6b13146 • Publication Date (Web): 22 Feb 2017

Downloaded from http://pubs.acs.org on February 24, 2017

\section{Just Accepted}

"Just Accepted" manuscripts have been peer-reviewed and accepted for publication. They are posted online prior to technical editing, formatting for publication and author proofing. The American Chemical Society provides "Just Accepted" as a free service to the research community to expedite the dissemination of scientific material as soon as possible after acceptance. "Just Accepted" manuscripts appear in full in PDF format accompanied by an HTML abstract. "Just Accepted" manuscripts have been fully peer reviewed, but should not be considered the official version of record. They are accessible to all readers and citable by the Digital Object Identifier (DOI®). "Just Accepted" is an optional service offered to authors. Therefore, the "Just Accepted" Web site may not include all articles that will be published in the journal. After a manuscript is technically edited and formatted, it will be removed from the "Just Accepted" Web site and published as an ASAP article. Note that technical editing may introduce minor changes to the manuscript text and/or graphics which could affect content, and all legal disclaimers and ethical guidelines that apply to the journal pertain. ACS cannot be held responsible for errors or consequences arising from the use of information contained in these "Just Accepted" manuscripts. 


\section{INTRODUCTION}

Protein engineering enables the design and synthesis of monodisperse polymers with functional domains drawn from nature or created de novo. ${ }^{1}$ Because protein polymers are made by expression of artificial genes, they can be modified easily and systematically by editing of their DNA coding sequences. In this manner, proteins have been engineered with binding domains that drive them to self-assemble into physically crosslinked networks. ${ }^{2}$ The non-covalent nature of domain association in these networks permits the constituent proteins to exchange binding partners. Such processes are common in polymeric systems; for example, block copolymer micelles in solution exchange chains at rates that are highly dependent on the architectures of the individual blocks, ${ }^{3-4}$ and telechelic polymers with hydrophobic endgroups form micellar networks that relax via chain disengagement from interconnected micelles. ${ }^{5}$ Exchange of polymeric strands also plays essential roles in biological processes, including repair of double-stranded DNA breaks by homologous recombination. ${ }^{6-7}$

Strand exchange dynamics are particularly important in governing the viscoelastic properties of hydrogels assembled from proteins that carry amphipathic $\alpha$-helical domains. ${ }^{2,8-9}$ Amphipathic helices are ubiquitous in nature, and often function by driving protein aggregation through the formation of coiled-coil bundles. ${ }^{10-12}$ Hydrogels assembled from coiled-coil proteins are reversible: they can disassemble and reassemble rapidly in response to external stimuli such as temperature changes or mechanical shear. ${ }^{2}$ These hydrogels are also shear thinning, injectable and potentially useful for delivery of cellular or molecular therapeutics. ${ }^{13}$ Because strand exchange underlies the physical behavior of the network, tuning the strand exchange rate is essential for optimizing hydrogel performance.

Characterization of strand exchange in coiled-coil systems has largely been limited to chromatographic analyses of equi- librium solutions, ${ }^{10,14-15}$ stopped-flow spectroscopy, ${ }^{16}$ and fluorescence dequenching experiments. ${ }^{9}, 17$ These techniques are most useful for analysis of dilute solutions, and cannot be applied directly to hydrogels. In contrast, fluorescence recovery after photobleaching (FRAP) is routinely used to assess macromolecular diffusion and binding in crowded environments such as the cellular milieu. ${ }^{18}$ For example, FRAP has been used to measure rates of binding of leucine-zipper transcription factors to chromatin in live cells. ${ }^{19}$ The method requires only minor perturbation of the system of interest through sparse labeling with fluorescent dyes, and is amenable to analysis by models that permit simultaneous determination of diffusion coefficients and binding constants. ${ }^{18,20}$ Although FRAP has been used to probe chain mobility in polymer networks, strand exchange has either not been important in these systems (e.g. in covalently crosslinked networks), ${ }^{21-22}$ or has not been quantified. ${ }^{23-26}$ The technique is commonly used only to estimate effective chain diffusivity, and when interchain binding is present, it is typically assessed qualitatively.

This report describes the use of FRAP to characterize the interplay between strand exchange and chain mobility in associative protein hydrogels. The gels were formed from an engineered triblock protein (designated "PEP") composed of two identical coiled-coil domains ("P") at the N- and C-termini, flanking a water-soluble midblock ("E") consisting of elastinlike polypeptide repeats (Supporting Information Table S1). The $\mathrm{P}$ domain is derived from the $\mathrm{N}$-terminal fragment of rat cartilage oligomeric matrix protein (COMP), and has been reported to form homopentameric coiled-coil bundles. ${ }^{8,27}$ Association of the $\mathrm{P}$ domains drives the reversible assembly of PEP into optically transparent, physically crosslinked networks. The viscoelastic behavior of PEP networks is analogous to that of other networks assembled by association of coiled-coil domains. ${ }^{13}$ 
Here we use FRAP to determine diffusion coefficients and equilibrium binding constants of fluorescently labeled PEP chains in PEP hydrogels. We find that the mobility of PEP chains is significantly reduced by reversible network association. To gain insight into the mechanism of chain mobility, we elaborate a previously developed 2-state reaction-diffusion model for FRAP into a 3-state "hopping" model of chain migration. ${ }^{18,20} \mathrm{We}$ find experimentally and in coarse-grained Brownian Dynamics simulations of gel-forming telechelic polymers that binding of one of the $\mathrm{P}$ domains in PEP reduces binding of the second. Finally, we show that tracer chain mobility is highly sensitive to structural changes in the coiled-coil endblocks. Taken together, our results furnish a new framework for understanding and controlling chain mobility in reversible polymer networks.

\section{RESULTS AND DISCUSSION}

Reversible PEP hydrogels show fluorescence recovery after photobleaching. To probe chain mobility in PEP hydrogels, we generated a series of fluorophore-labeled probes that would associate with network junctions in a defined manner, without affecting the rheological behavior of the network. We first performed site-directed mutagenesis on PEP to introduce a single cysteine residue into the elastin-like midblock, resulting in $\mathrm{PE}_{\mathrm{C}} \mathrm{P}$ (Table S1). The absence of other cysteines in the protein enabled site-specific conjugation of fluorescein-5-maleimide $(f 5 m)$ to the central thiol via Michael-type addition (Figure 1A and Figure $\mathrm{S} 1$ ). The $\mathrm{PE}_{\mathrm{C}} \mathrm{P}-f 5 m$ conjugate yielded homogeneous, fluorescent gels when added at low concentrations into PEP networks (typically $\mathrm{PE}_{\mathrm{C}} \mathrm{P}$ to PEP ratios of 1:50-100 were used). Using oscillatory shear rheometry, we verified that the rheological behavior of PEP gels was minimally perturbed by this labeling strategy (Figure S2).

We next prepared fluorescent PEP hydrogels of defined thickness $(\sim 120 \mu \mathrm{m})$, and photobleached cylindrical volumes in each gel using a standard confocal microscope. In $10 \%$ weightto-volume $(w / v)$ gels, we observed steady recovery of fluorescence intensity within the photobleached spot (Figure 1B). Fluorescence recovery results from diffusion of unbleached fluorophore into the photobleached region, and confirms that $\mathrm{PE}_{\mathrm{C}} \mathrm{P}$ chains are mobile within PEP networks. Consistent with our hypothesis that $\mathrm{PE}_{\mathrm{C}} \mathrm{P}$ is associated with the network, we observed accelerated rates of fluorescence recovery in networks solubilized with $8 \mathrm{M}$ urea, a common protein denaturant. We discuss each of these results in more detail below.

Quantitative analysis of chain mobility. Gels were prepared at protein concentrations ranging from $2 \%$ to $10 \% \mathrm{w} / \mathrm{v}$ (gelation in PEP solutions occurs near 3\%). As expected, the rate of fluorescence recovery after photobleaching decreased with increasing protein concentration (Figure 2A). To quantify chain mobility, we fit the experimental FRAP curves to a model that attributes fluorescence recovery to diffusion only (see Supporting Information, Equations 23, 25 and 30). Such an analysis is similar to standard FRAP analyses of diffusion in polymer networks $^{22-23,25-26,28}$, and results in a single parameter termed $D_{\text {eff }}$, the effective diffusion coefficient. ${ }^{18}$ In the case of PEP networks, fluorescence recovery represents diffusion slowed by binding; $D_{\text {eff }}$ provides a measure of the mobility of polymer chains for which Brownian motion is constrained by reversible network association. The effective diffusion model yielded good fits to the fluorescence recovery curves (Figure 2), enabling us to estimate $D_{\text {eff }}$ for each gel. $D_{\text {eff }}$ decreases steeply with

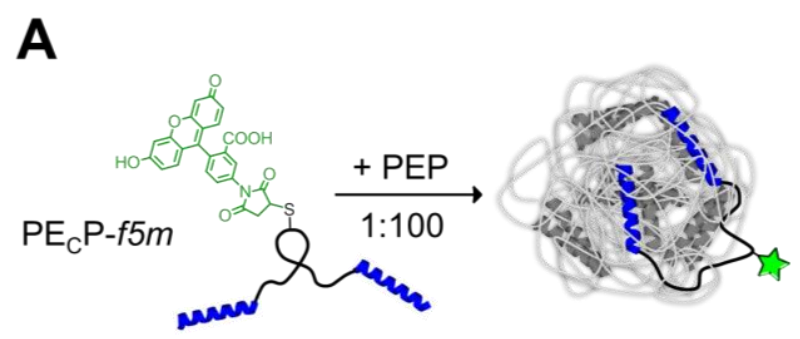

B
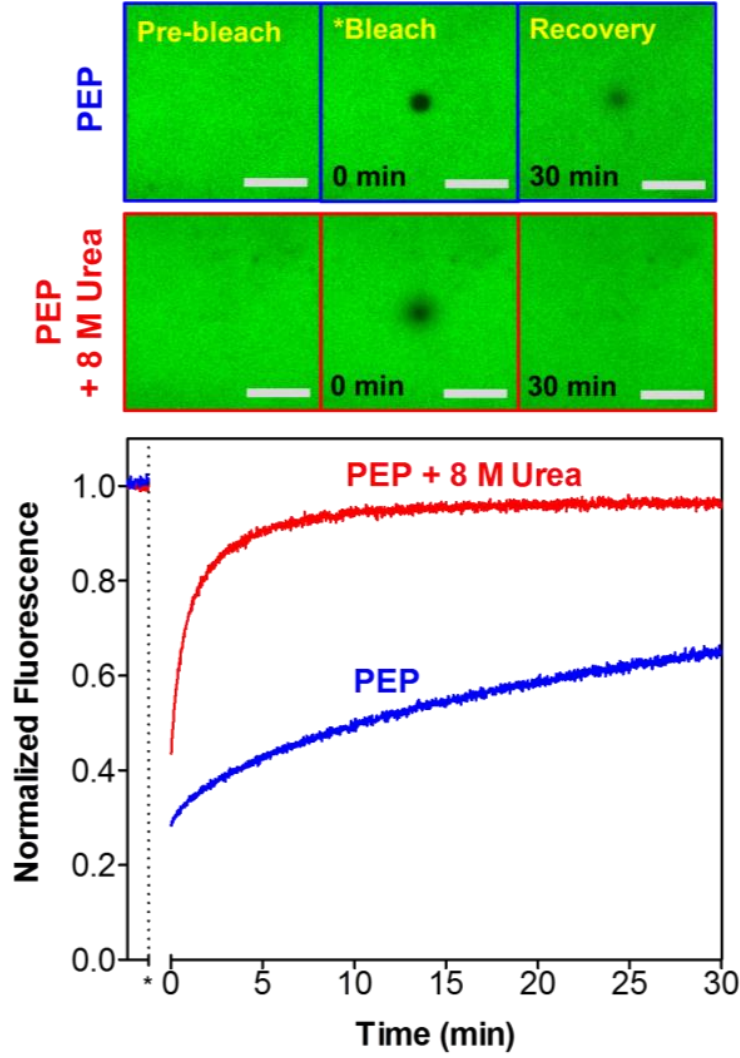

Figure 1. Fluorescence recovery after photobleaching in labeled PEP hydrogels. (A) Labeling of PEP hydrogels was achieved by addition of a fluorescent PEP analogue ( $\mathrm{PE}_{C} \mathrm{P}-\mathrm{f} 5 \mathrm{~m}$ ) at low concentrations. (B) FRAP in 10\% $w / v$ PEP hydrogels as monitored by confocal microscopy. A circular bleach spot with a radius (a) of 12.5 $\mu \mathrm{m}$ recovers slowly over a period of $30 \mathrm{~min}$ (blue curve). The same network solubilized in $8 \mathrm{M}$ urea shows accelerated fluorescence recovery (red curve). Scale bar $100 \mu \mathrm{m}$

increasing protein concentration, dropping from $1.3 \times 10^{-8} \mathrm{~cm}^{2}$ $\mathrm{s}^{-1}$ in viscous $2 \%$ solutions to $2.3 \times 10^{-10} \mathrm{~cm}^{2} \mathrm{~s}^{-1}$ in $10 \%$ gels (Figure 2B).

We attribute the slower recovery at higher protein concentrations primarily to the increased concentration of binding sites, although changes in network topology such as loop suppression and chain entanglement may also suppress chain release from junctions. ${ }^{5,8,29}$ To test whether the effective diffusivity is primarily controlled by reversible endblock binding, we measured chain mobility as a function of the concentration of the protein denaturant urea. At a fixed protein concentration of $10 \%$, the rate of fluorescence recovery increased abruptly with increasing concentration of urea (Figure 2C); addition of $2 \mathrm{M}$ urea increases $D_{\text {eff }} 9$-fold (Figure 2D). The abruptness of the change suggests that modest concentrations of urea are sufficient to inhibit association of the $\mathrm{N}$ - and $\mathrm{C}$-terminal domains of PEP. 


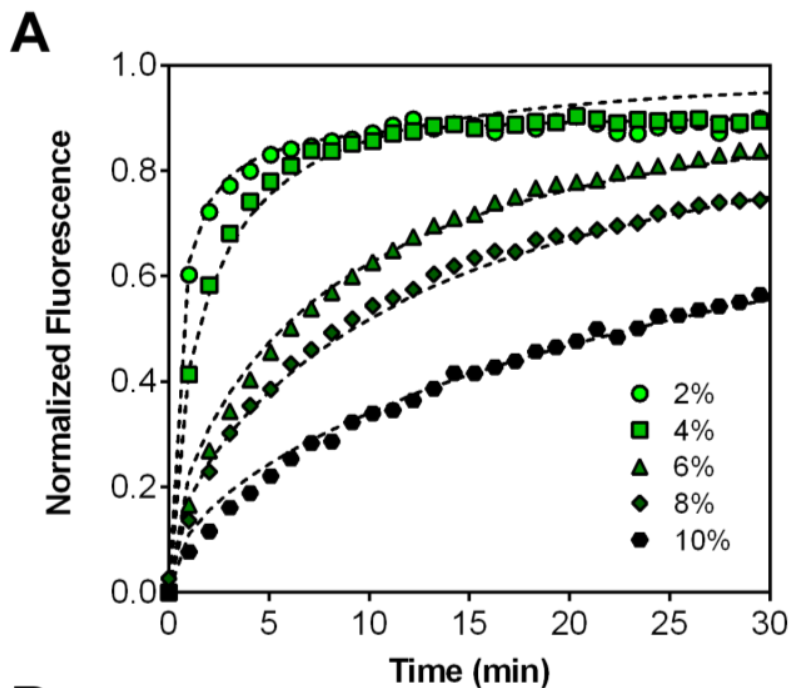

B

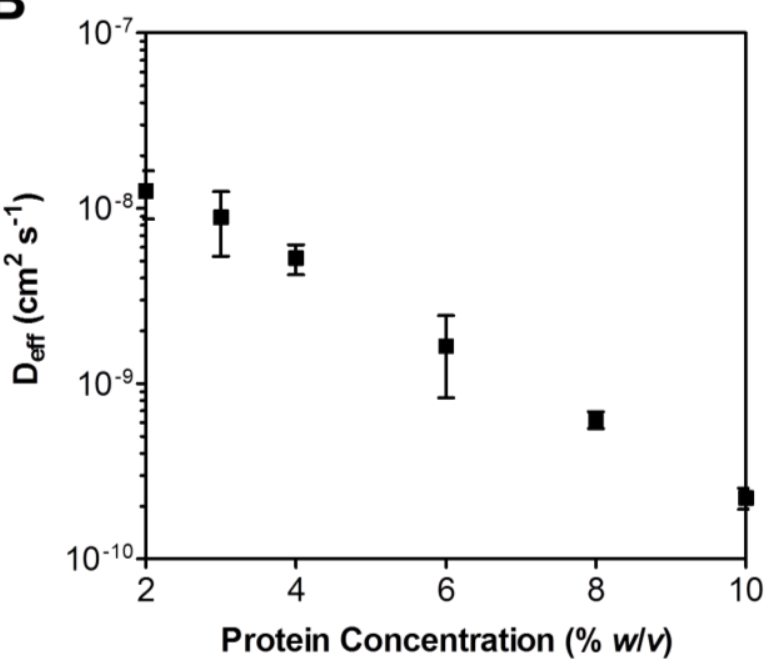

D

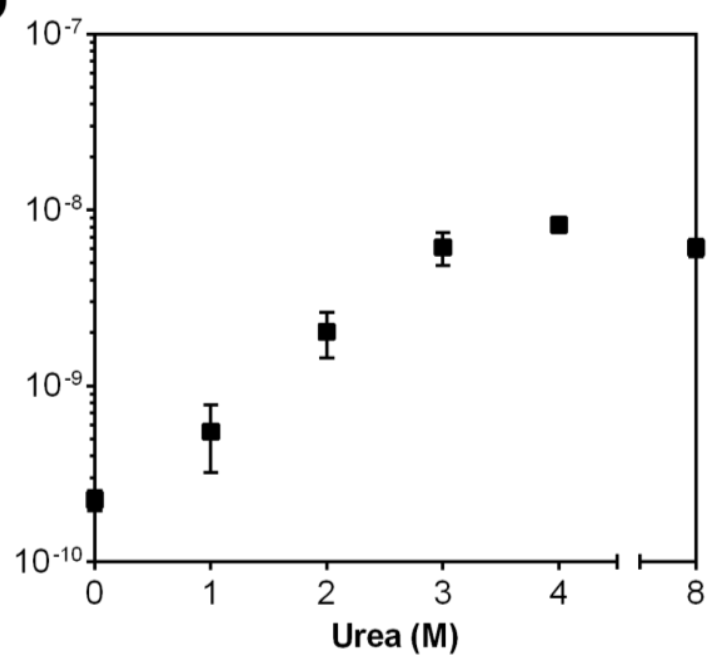

Figure 2. The rate of fluorescence recovery after photobleaching in PEP hydrogels depends on gel density and concentration of denaturant. (A) FRAP curves generated from gels prepared at protein concentrations ranging from $2 \%$ to $10 \%$, showing that the recovery rate decreases with increasing gel density. (B) Quantification of effective chain mobility as a function of gel density. $D_{e f f}$ varies inversely with gel density. (C) FRAP curves generated from $10 \%$ protein solutions prepared in increasing concentrations of urea. Fluorescence recovery rates increase with increasing amounts of urea, indicating disruption of interchain binding. (D) Quantification of the urea recovery curves. $D_{\text {eff }}$ rises with increasing concentrations of urea, eventually reaching a plateau above $3 \mathrm{M}$. Error bars represent mean \pm standard deviation $(n \geq 3$ recovery curves from at least two gels). Dashed curves in A and B represent fits generated from the effective diffusion model.

Disruption of interchain binding destroys network integrity; samples prepared in high concentrations of urea (greater than 2 M) were viscous liquids.

A 3-state "hopping” model of chain migration in reversible hydrogels. Although the preceding analysis provides a useful description of chain mobility in PEP networks, it does not separate the effects of diffusion and interchain association. We sought to distinguish the roles of diffusion and binding in PEP networks. To this end, we formulated a model that captures both the diffusive and reactive elements of strand exchange in a physical molecular network. Our model is an extension of a 2 state reaction-diffusion model originally developed by Sprague et al., which relates the rate of fluorescence recovery to an equilibrium between two states: one free and one bound. ${ }^{18}$ Because each PEP chain has two terminal P domains, we chose to model network association as an equilibrium involving three sequential states (represented schematically in Figure 3A):

$$
f \stackrel{K_{1}}{\rightleftharpoons} d \stackrel{K_{2}}{\rightleftharpoons} b
$$

In the free state $(f)$ neither $\mathrm{P}$ domain is bound to another and the chain can diffuse throughout the network with a self-diffusivity $D_{f}$. If both $\mathrm{P}$ domains on the chain join coiled-coil bundles, the chain enters the bound state $(b)$ and becomes fully network-associated. We also consider an intermediate dangle state (d) in which only one of the P domains is tethered to the network. We distinguish the diffusion coefficient of free chains $D_{f}$ from the effective diffusion coefficient $D_{\text {eff }}$, which represents diffusion slowed by binding. Because $D_{f}$ represents free diffusion in the absence of binding, $D_{\text {eff }}$ will be smaller than $D_{f}$ whenever binding interactions are significant.

We make several simplifying assumptions that will be considered further below. First, we assume that both binding processes ( $f \rightleftharpoons d$ and $d \rightleftharpoons b$ ) achieve equilibrium, and that both are governed by the same equilibrium constant $\left(K_{1} \approx K_{2}=\right.$ 
A
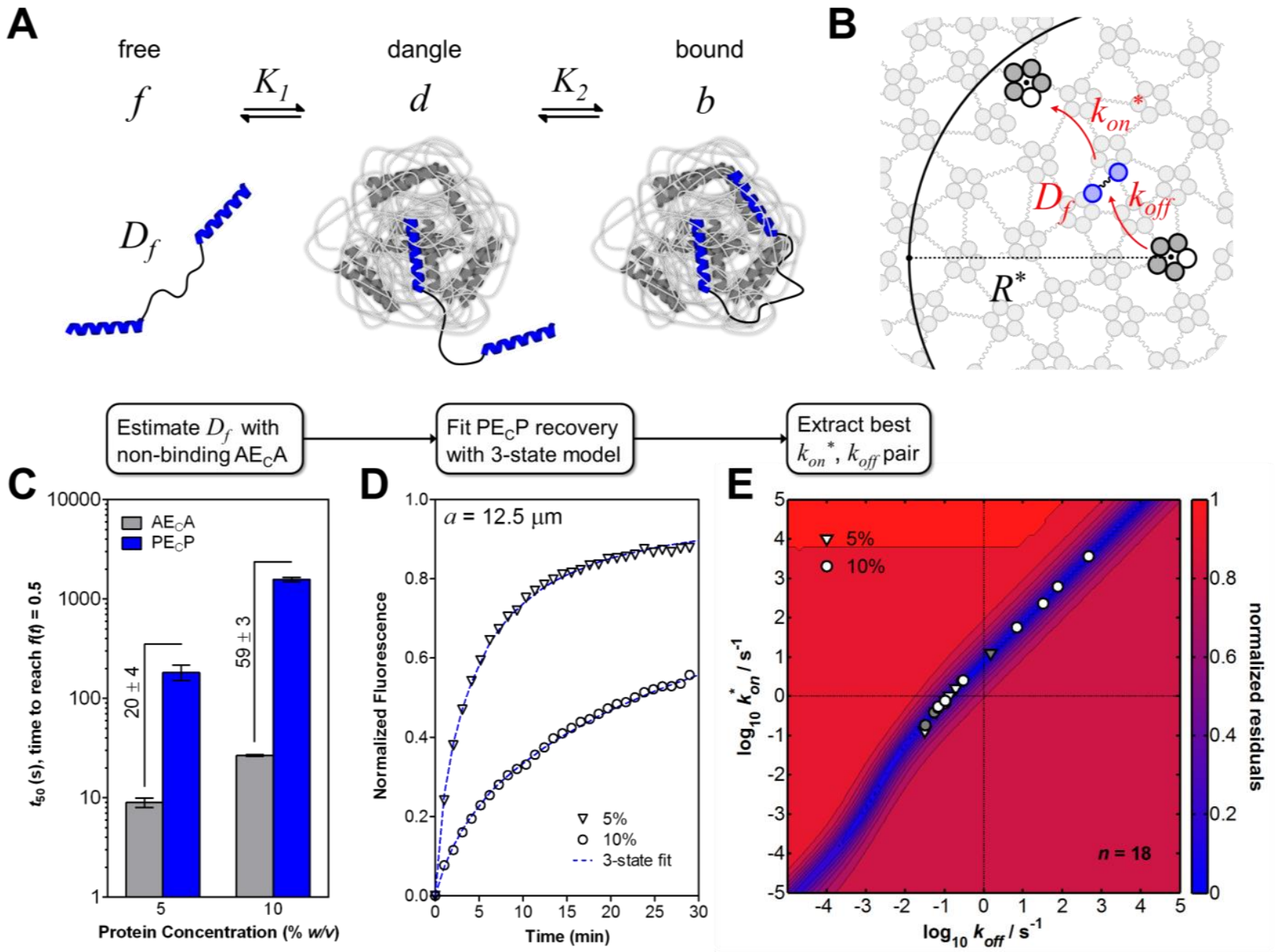

Figure 3. A reaction-diffusion analysis of chain migration in reversible hydrogels. (A) Illustration of the 3-state model. Chains transition between free $(f)$, dangle $(d)$ and bound $(b)$ states, and can diffuse only in the free state. (B) After a chain dissociates from an initial binding site (at a rate determined by $k_{o f f}$ ), it reassociates with a new junction at a rate determined by $k_{o n}{ }^{*}$. The average distance a free chain diffuses ("hops") before rebinding is $R^{*}$. (C) $\mathrm{AE} C \mathrm{~A}$, a non-binding probe without terminal coiled-coils shows rapid fluorescence recovery compared to the associative probe $\mathrm{PE}_{\mathrm{C}} \mathrm{P}$ (vertical text shows fold-change \pm standard deviation, $n=3$ recovery curves measured in one gel preparation for each probe). (D) The 3-state model yields excellent fits to the normalized recovery curves for a bleach spot radius (a) of $12.5 \mu \mathrm{m}$. (E) Contour map showing normalized residuals of a representative 3 -state model fit to a recovery curve from a $10 \%$ gel $(a=12.5 \mu \mathrm{m})$ for a wide range of $k_{o n}{ }^{*}$ and $k_{o f f}$ values. Points on the map represent $\left(k_{o n}{ }^{*}, k_{o f f}\right)$ pairs obtained from independent photobleaching experiments performed in multiple gels $(a=12.5 \mu \mathrm{m}, n=12)$. Shaded symbols are experiments performed with a larger spot size $(a=25 \mu \mathrm{m}, n=6)$. The values of $k_{o n}{ }^{*}$ obtained from 5\% gels were multiplied by 2 in order to compare them with values from 10\% gels on the same map.

$\left.k_{o n}{ }^{*} / k_{o f f}\right)$. Note that ${k_{o n}}^{*}=k_{o n} S_{e q}$ is a pseudo-first-order rate constant calculated from the true association rate constant $k_{o n}$ (a second-order rate constant) by assuming a constant concentration of binding sites $S_{\text {eq }}{ }^{18}$ We also assume that each P domain has a single binding mode, and that chain mobility in either of the two associated states $(d$ or $b)$ is negligible $\left(D_{d}=D_{b} \approx 0\right)$. The physical picture is therefore one in which chains are constrained to migrate by "hopping" from site to site, but are otherwise fixed in space (Figure 3B). The distance a chain travels during such a transition (the "hopping radius") is:

$$
R^{*}=\sqrt{\frac{6 D_{f}}{k_{o n}^{*}}}
$$

A material balance on eq. 1 results in a system of three coupled reaction-diffusion equations that can be used to model experimental FRAP curves and to estimate the three parameters in the model $\left(k_{o n}{ }^{*}, k_{\text {off }}\right.$ and $\left.D_{f}\right)$. We sought an analytical solution to the 3-state reaction-diffusion model. Following Sprague et al. for the 2-state model, ${ }^{18}$ Laplace transformation of eq. 1 yielded an analytical solution involving modified Bessel functions in Laplace space (see Figures S3, S4 and Supporting Information for details). When binding is neglected $\left(k_{o n}{ }^{*} \rightarrow 0\right.$ and $\left.k_{\text {off }} \rightarrow \infty\right)$, the new solution reduces to the previously reported closed form solution for free diffusion in a circular bleach spot. ${ }^{18,30}$ Numerical inversion of the Laplace-domain solution using the MATLAB routine invlap. $m$ produces the time-domain response,$^{31}$ providing estimates of model parameters by comparison with experimental curves. 
FRAP curves simulated using the 3-state model were fit to experimental curves using the MATLAB routine nlinfit.m, as well as a custom curve fitting algorithm that gave comparable results (Figure S5). With this approach, we found it difficult to obtain reliable estimates of all three model parameters from a single curve. Therefore, we simplified our curve-fitting procedure by first estimating $D_{f}$ in a separate FRAP experiment using a non-binding elastin-like probe where the $\mathrm{P}$ domain endblocks were replaced by an irrelevant " $\mathrm{A}$ " peptide that does not form coiled-coils (see Table $\mathrm{S} 1$ for sequence). ${ }^{32-33}$

Recovery rates observed with the non-binding " $\mathrm{AE} \mathrm{C}_{\mathrm{C}} \mathrm{A}$ " probe were 20- to 50-fold faster than those observed with the $\mathrm{PE}_{\mathrm{C}} \mathrm{P}$ probe (Figure $3 \mathrm{C}$ and Figure S6). This provides further evidence that chain mobility is substantially reduced by reversible association of the coiled-coil domains. By attributing the recovery of $\mathrm{AE}_{\mathrm{C}} \mathrm{A}$ to diffusion alone, we estimated that $D_{f}$ for an unbound PEP chain is approximately $1.59 \times 10^{-8} \mathrm{~cm}^{2} \mathrm{~s}^{-1}$ in a $10 \%$ gel (assuming $D_{f} \sim M^{-3 / 5}$ for a polymer chain in good solvent). ${ }^{28}$ This value is similar to $D_{\text {eff }}$ in dilute solutions of PEP (Figure $2 \mathrm{C}$ ), and is within range of the diffusivities reported for macromolecules in other hydrogels. For example, dextran probes of similar molecular weight diffuse through dextran solutions and gels at approximately $10^{-7} \mathrm{~cm}^{2} \mathrm{~s}^{-1}$, and unbound globular proteins diffuse through poly(ethylene glycol) gels at rates of $10^{-7}$ $-10^{-9} \mathrm{~cm}^{2} \mathrm{~s}^{-1}$, depending on the hydrodynamic radius of the protein and the mesh size of the network. ${ }^{22,28,34-36}$

Next we sought to estimate $k_{\text {on }}{ }^{*}$ and $k_{\text {off }}$ for $\mathrm{PE} \mathrm{C}$, using a value of $1.59 \times 10^{-8} \mathrm{~cm}^{2} \mathrm{~s}^{-1}$ for $D_{f}$ in the 3 -state model. Following Sprague et al., a grid of all possible $\left(k_{o n}{ }^{*}, k_{\text {off }}\right)$ pairs was sampled in log space (typically in increments of $10^{0.1}$ between $10^{-5}$ and $10^{5} \mathrm{~s}$ ) in order to find the pair that minimized the residuals between the simulated and experimental curves. This pair was then supplied as the initial guess in the MATLAB algorithm nlinfit.m, which finally produced a unique $\left(k_{o n}{ }^{*}, k_{\text {off }}\right)$ pair corresponding to the best fit. ${ }^{18}$ Excellent fits to experimental FRAP curves were obtained with this procedure (Figure 3D). Within the range of bleach spot radii that we explored $(a=1-25 \mu \mathrm{m})$, the quality of the fit was relatively insensitive to the individual values of the rate constants, but strongly dependent on their ratio (Figure 3E). For a 10\% gel and spot radius of $12.5 \mu \mathrm{m}$, the data lie along a line with slope $k_{\text {on }}{ }^{*} / k_{\text {off }}=7.4 \pm 0.9$, whereas $k_{\text {on }}{ }^{*}$ itself ranges from $0.2 \mathrm{~s}^{-1}$ to $3.6 \times 10^{3} \mathrm{~s}^{-1}$.

To obtain estimates of the individual values of the rate constants, we made the assumption that $k_{\text {off }}$ corresponds to the network relaxation rate measured by oscillatory shear rheometry (Figure S2, $k_{\text {off }} \approx \omega_{\mathrm{c}}$ ), and used the ratio of $k_{\text {on }}{ }^{*}$ to $k_{\text {off }}$ to obtain $k_{o n}{ }^{*}$. This provides $k_{\text {off }}=0.51 \pm 0.02 \mathrm{~s}^{-1}$ and $k_{\text {on }}{ }^{*}=3.8 \pm 0.5 \mathrm{~s}^{-1}$, suggesting a relatively weak binding equilibrium for the $\mathrm{P}$ domain. Strand exchange rates $\left(k_{\text {off }}\right)$ reported for coiled-coils vary widely, e.g., $3 \times 10^{-3} \mathrm{~s}^{-1}\left(\mathrm{GCN}_{4}\right), 373 \times 10^{-4} \mathrm{~s}^{-1}-0.7 \mathrm{~s}^{-1}$ (model leucine zippers), ${ }^{16} 0.2 \mathrm{~s}^{-1}$ (Fos/Jun), ${ }^{38} 1 \times 10^{-4} \mathrm{~s}^{-1}$ ( $\alpha$-tropomyo$\sin ),{ }^{39} 6 \times 10^{-7} \mathrm{~s}^{-1}-5 \times 10^{-3} \mathrm{~s}^{-1}$ (4-helix bundle proteins)..$^{9,}{ }^{40-41}$ Refolding and association rates $\left(k_{o n}{ }^{*}\right)$ are typically much faster (e.g., for Fos/Jun and GCN4, roughly $1 \mathrm{~s}^{-1}$ even at low $\mu \mathrm{M}$ concentrations, resulting in dissociation-limited exchange kinetics with $K_{d}$ on the order of $0.01-1 \mu \mathrm{M}$ for these zippers). ${ }^{16,38,42}$ By comparison, all fits in Figure 3E give an average dissociation constant of $K_{d}=173 \pm 29 \mu \mathrm{M}$. This leads to a free energy of network association $\Delta G_{a}=-5.1 \pm 0.1 \mathrm{kcal} \mathrm{mol}^{-1}$. This number is within range of the Gibbs free energy of pentamer formation for native $\mathrm{P}\left(\Delta G^{\circ}=-4.3 \mathrm{kcal} \mathrm{mol}^{-1}\right)$ estimated from thermal denaturation curves using circular dichroism spectroscopy, and is similar in magnitude to folding energies for other weakly associating coiled-coil structures. ${ }^{43-44}$

Sprague et al. showed that, for the 2-state reaction-diffusion model, the full model may be simplified to the single-parameter effective diffusion model (i.e., $D_{\text {eff }}$ alone gives good fits) whenever the dimensionless constant $k_{o n}{ }^{*} a^{2} / D_{f}$ is significantly greater than unity. ${ }^{18}$ This constraint ensures that binding is rapid relative to the characteristic diffusion time of the experiment. An important characteristic of this regime is that the rate of fluorescence recovery is insensitive to the individual values of $k_{o n}{ }^{*}$ and $k_{\text {off }}$, and depends only on their ratio. Using the above estimates for $k_{o n}{ }^{*}$ and $D_{f}$, we find that $k_{o n}{ }^{*} a^{2} / D_{f} \sim 10^{2}$ when $a=12.5 \mu \mathrm{m}$. This suggests that all of the FRAP experiments reported here lie in the effective diffusion regime. This explains the imprecision in the estimates of ${k_{o n}}{ }^{*}$ and $k_{\text {off }}$ derived from our FRAP experiments (Figure 3E), and our ability to generate good fits of our FRAP curves using $D_{\text {eff }}$ alone (Figure $2 \mathrm{~A}$ ).

Predicting the hopping mobility with the 3-state model. Given that $k_{\text {on }}{ }^{*} a^{2} / D_{f} \gg 1$ (see the above discussion), we can assume local equilibrium during the fluorescence recovery process. Under this assumption, it can be shown that for a chain with $N$ associative domains (i.e., for an " $N+1$ "-state hopping model, see Supporting Information, Equations 31-37), the ratio $D_{f} / D_{\text {eff }}$ is given by

$$
\frac{D_{f}}{D_{e f f}}=1+K_{1}+K_{1} K_{2}+\cdots+\prod_{i=1}^{N} K_{i}
$$

This allows us to predict the hopping mobility $D_{\text {eff }}$ for a chain with any number of associative domains, provided $D_{f}$ and the equilibrium constants are known. In the case of the 3-state model (eq. $3, N=2$ ) if only one of the equilibrium constants is known, it is possible to make inferences about the relative magnitudes of $K_{1}$ and $K_{2}$ by comparing predictions from eq. 3 to experimental mobilities. We therefore designed a " $\mathrm{PE}_{\mathrm{C}}$ " probe that could associate with the network only once. The recovery curve of $\mathrm{PE}_{\mathrm{C}}$ should reflect the equilibrium between free and dangling chains, thus providing an independent measurement of $K_{1}$. We also refined our estimates of $D_{f}$ by measuring the recovery rate of a non-binding " $E_{C}$ " probe comprising only the elastin-like midblock. As before, we assume $D_{f} \sim M^{-3 / 5}$ in order to estimate $D_{f}$ for the larger, associative probes.

The fluorescence recovery curves for these probes are shown in Figure 4A. From the $\mathrm{E}_{\mathrm{C}}$ probe we estimated $D_{f}$ for $\mathrm{PE}_{\mathrm{C}} \mathrm{P}$ as $2.94 \pm 0.35 \times 10^{-8} \mathrm{~cm}^{2} \mathrm{~s}^{-1}$. This is roughly 2 -fold larger than the value estimated from the recovery rate of $\mathrm{AE}_{\mathrm{C}} \mathrm{A}$, and suggests a slight tendency for the A domain to self-associate. Fitting the $\mathrm{PE}_{\mathrm{C}}$ recovery with a 2-state model (eq. 3, $N=1$ ) provides $K_{1}=$ $26.5 \pm 4.5$. Under the assumption that $K_{1}=K_{2}$, this estimate can be applied directly to the 3 -state model (eq. 3, $N=2$ ) in order to predict $D_{\text {eff }}$ for $\mathrm{PE}_{\mathrm{C}} \mathrm{P}$. This approach substantially under-predicts the observed mobility (Figure 4B, $D_{o b s}=5.1 \times D_{\text {pred }}$ ). Moreover, fitting the $\mathrm{PE}_{\mathrm{C}} \mathrm{P}$ recovery with a 3-state model without prior knowledge of $K_{1}$ (again assuming equivalence of $K_{1}$ and $K_{2}$ ) provides $K_{1}=K_{2}=11.7 \pm 1.8$. These data are summarized in Table 1.

We hypothesized that the disparity in the values of $K_{1}$ obtained from the $\mathrm{PE}_{\mathrm{C}}$ and $\mathrm{PE}_{\mathrm{C}} \mathrm{P}$ probes might reflect a difference in the values of the equilibrium constants for sequential binding of the two $\mathrm{P}$ domains of $\mathrm{PE}_{\mathrm{C}} \mathrm{P}$ (Figure $3 \mathrm{~A}$ ), with $K_{l}$ greater than $K_{2}$. To test this hypothesis, we performed coarse-grained Brownian Dynamics simulations of gel-forming telechelic 
A

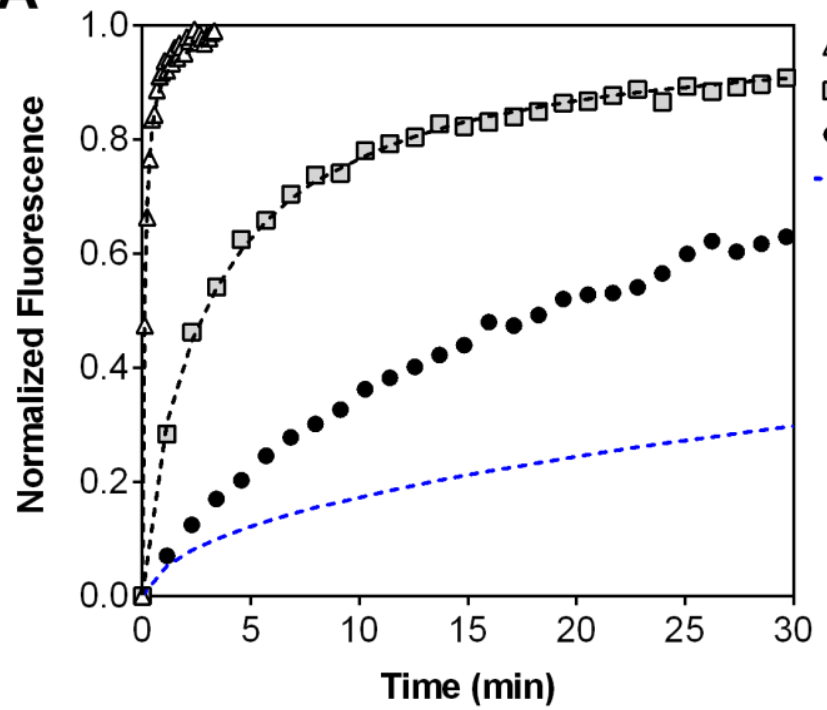

B

$\Delta E_{C}$

$\square P E_{C}$

- $P E_{C} P$ obs

-.. $P E_{C} P$ pred

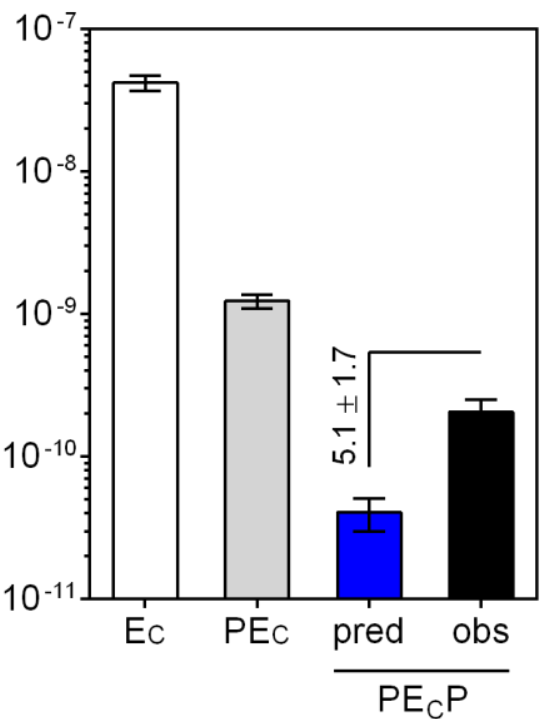

C

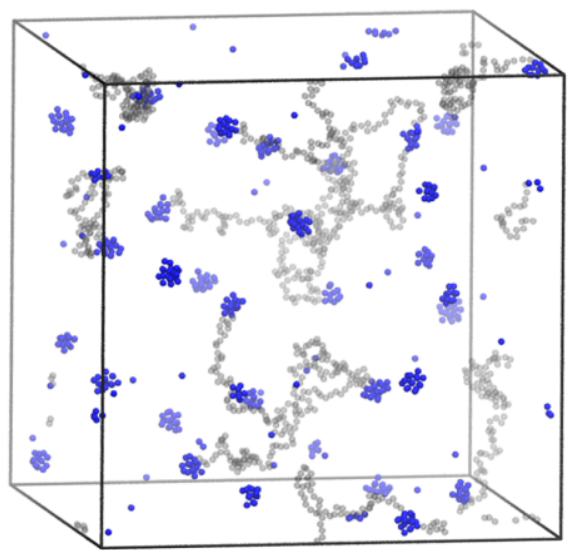

D

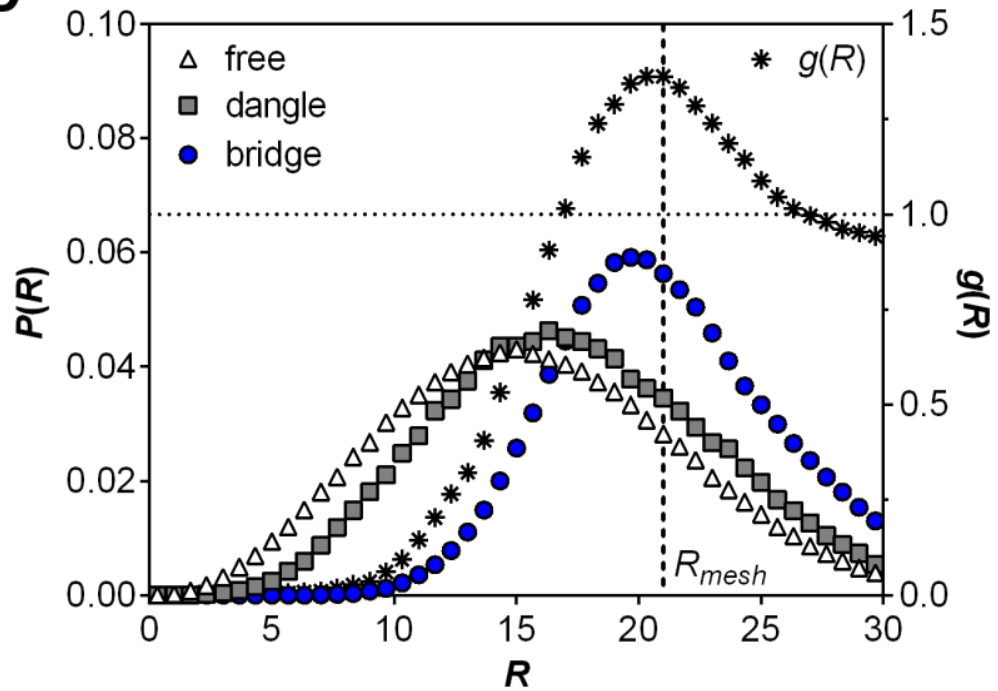

Figure 4. Predictions from the 3-state model imply binding asymmetry in PEP hydrogels. (A) FRAP experiments on $E_{C}$ and PE $E_{C}$ probes provide independent estimates of $D_{f}$ and $K_{l}$ that, together with eq. (3), predict $D_{\text {eff }}$ and the recovery rate of PECP (blue dashed line). The experimentally observed recovery rate is higher than predicted, suggesting asymmetric sequential binding where $K_{l}>K_{2}$. Fits to the EC and $\mathrm{PE}_{\mathrm{C}}$ curves were generated with 1 -state (eq. 3, $N=0$ ) and 2-state (eq. 3, $N=1$ ) effective diffusion models, respectively (black dashed lines). (B) Assuming $K_{l}=K_{2}$ under-predicts the observed $D_{\text {eff }}$ for PECP by roughly 5 -fold (mean $\pm \mathrm{SD}, n \geq 2$ gel preparations per probe). (C) Snapshot of a simulated gel with stickers (blue) connected by non-sticker beads (grey). The non-sticker beads of only 10 chains are shown for clarity. (D) Origin of the binding asymmetry. The radial distribution function of network junctions $g(R)$ is shown together with the chain end-to-end distributions $P(R)$ for the three states (bins of $\Delta R=0.67$ were used in computing the distributions). Free and dangling chains can adopt a substantial set of conformations at distances $R<R_{m e s h}$, the location of maximum junction density. These conformations are lost upon entry into the bridge state.

polymers (see Supporting Information for details). We used a Kremer-Grest bead-spring model with "sticky" beads at the chain ends interacting through an attractive Lennard-Jones potential. ${ }^{45}$ Figure $4 \mathrm{C}$ shows a representation of a gel comprised of chains with a length of 100 beads. The stickers cluster to form distinct network junctions, which we define as groups of neighboring stickers. By analogy to the 3-state model, we define the state of a simulated chain by specifying whether its stickers are both free from junctions $(f)$ or both attached to junctions $(b)$, or if only one sticker is bound $(d) . K_{l}$ and $K_{2}$ are then obtained by computing the fraction of chains in each state.
We find that a majority of the chains in our simulation are fully bound ( $[b]_{e q}=0.86$, see also Figure S7), in good agreement with the fraction of bound PEP chains estimated by FRAP (Table $\left.1,[b]_{e q}=0.91\right)$. Importantly, asymmetry in the two binding constants is apparent in the simulation, with $K_{1}=21.2, K_{2}=6.3$, and $K_{1} / K_{2}=3.4$. We can also isolate $K_{1}$ and $K_{2}$ from our FRAP data by assigning the $k_{o n}{ }^{*} / k_{\text {off }}$ ratio obtained from $\mathrm{PE}_{\mathrm{C}}$ to $K_{1}$, and then resolving the discrepancy between $D_{\text {pred }}$ and $D_{o b s}$ for $\mathrm{PE}_{\mathrm{C}} \mathrm{P}$ by treating $K_{2}$ as an adjustable parameter (Supplementary Equation 37). Interpreting the FRAP data in this way provides $K_{1}=$ $26.5 \pm 4.5, K_{2}=6.0 \pm 2.1$, and $K_{1} / K_{2}=4.4 \pm 1.7$, in good agreement with the simulation. These observations are consistent 

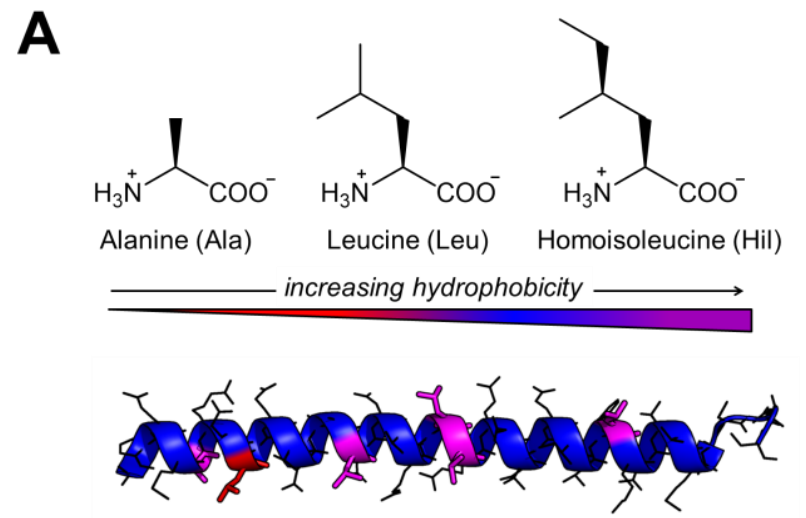

APQMLRE LQETNAA LQDVREL LRQQVKE ITFLKNT VMESDAS abcdefg \L37A

B

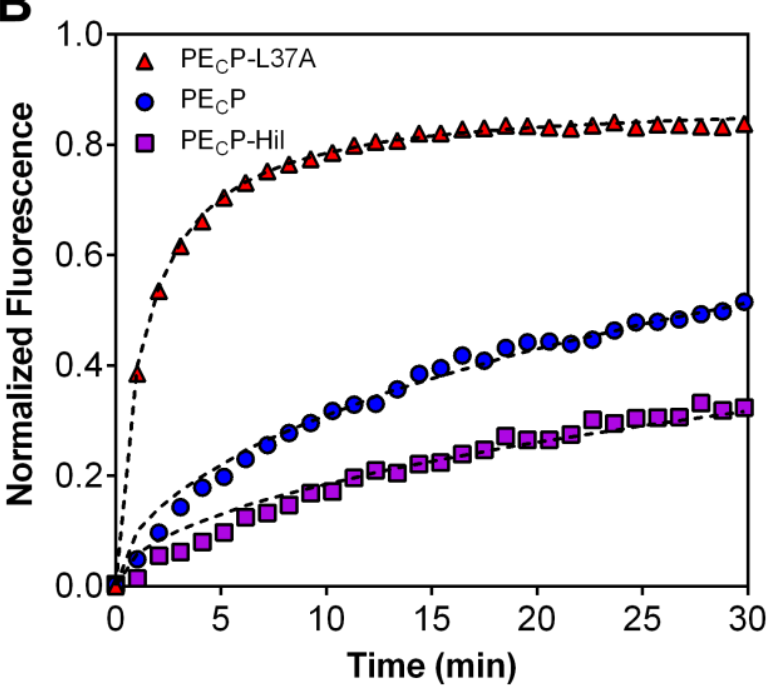

Figure 5. Genetic manipulation of the $\mathrm{P}$ domain controls the effective mobility of $\mathrm{PE}_{\mathrm{C}} \mathrm{P}$ probes. (A) PyMOL rendering of a single $\mathrm{P}$ domain $\alpha$-helix showing the location of key Leu residues (purple). An Ala mutation at position 37 (red) is known to destabilize binding, and was predicted to increase probe mobility. Global replacement of Leu with the non-canonical amino acid Hil was predicted to increase the hydrophobic surface area of the probe and decrease its mobility. (B) FRAP of the engineered probes. PECP-L37A shows accelerated fluorescence recovery relative to $P_{C} P$, whereas $\mathrm{PEcP}-\mathrm{Hil}$ shows slower recovery. Dashed lines depict fits generated from the effective diffusion model

with the hypothesis that reversible binding of a telechelic polymer to a macromolecular network is inherently asymmetric: the second binding event is disfavored relative to the first.

We propose that the inequality of $K_{1}$ and $K_{2}$ arises from a difference in the entropic penalties associated with successive binding events. In transitioning from the free to the dangle state, a chain becomes restricted to a fraction of the system volume, and loses entropy in proportion to the change in accessible volume. The subsequent transition from dangle to bridge causes a similar entropic loss, but with the additional constraint that the volume accessible to the remaining chain end also depends on the junction spacing. Gelation promotes a depletion of neighboring junctions below the characteristic mesh size of the gel. Dangling chains must discard the rich set of conformations accessible below this length scale when they bridge neighboring junctions.
The effects of network structure on chain conformation are apparent in our simulation. Figure 4D compares the distributions of chain end-to-end distances $P(R)$ for the three major states to $g(R)$, the junction radial distribution function. Free and dangling chains can access a substantial set of conformations at distances $R<R_{m e s h}$, the location of maximum junction density. In contrast, bridged chains are restricted to a narrower set of end-to-end distances that correspond closely to $R_{\text {mesh }}$. Mild chain stretching in the bridged state is also apparent, which may enhance the degree of binding asymmetry we observe (the average end-to-end distance of bridged chains $R_{b}$ exceeds that of dangling chains $R_{d}$ by a factor of 1.2). However, substantial conformational freedom may still be lost in transition from dangle to bridge, even in the absence of chain stretching.

An intriguing possibility is that, in addition to hopping, the diffusivity of a $\mathrm{PE}_{\mathrm{C}} \mathrm{P}$ probe may be enhanced by "walking"; i.e., by cycling between the dangle and bound states $d$ and $b$. In this process, the chain migrates through the network in discrete steps that correspond to the average distance between binding sites. A simple scaling analysis argues that this diffusive mode is not significant in PEP gels. Consider a chain with both ends bound to the network. The characteristic diffusivity of this chain can be estimated as $D_{b} \sim R_{b}{ }^{2} / \tau_{b}$, where $\tau_{b} \approx k_{o f f}{ }^{-1}$ is the average lifetime of the bound state. The expected contribution of this state to $D_{e f f}$ is $[b]_{e q} D_{b}$. As before, we obtain $k_{o n}{ }^{*}$ and $k_{\text {off }}$ for each state by setting $k_{\text {off }}$ equal to the relaxation rate obtained from rheometry (Figure S2), then using the $k_{o n}{ }^{*} / k_{\text {off }}$ ratios calculated from FRAP (Table 1). Independent estimates of $R_{b}$ from Flory theory $\left(R \sim b N^{3 / 5}\right){ }^{28}$ light-scattering measurements on unstructured amino acid midblocks, ${ }^{46}$ and a geometric argument based on binding site density suggest $R_{b}=7.8-13.7 \mathrm{~nm}$ for an ideal PEP network. These estimates provide $[b]_{e q} D_{b} \approx 0.0023 D_{\text {eff }}$ for bound chains and $[d]_{e q} D_{d} \approx 0.0029 D_{\text {eff }}$ for dangling chains, whereas $[f]_{e q} D_{f} \approx 1.00 D_{\text {eff. }}$. Other modes of bound mobility, including diffusion of chains in large-scale clusters, are excluded by a similar analysis.

We can appreciate why hopping dominates the mobility of PEP chains by considering the hopping radius $R^{*}$ in relation to $R_{b} \approx R_{\text {mesh }}$. From eq. (2) we estimate that the average distance of a hop is $R^{*}=1100 \pm 240 \mathrm{~nm}$, roughly 100 -fold larger than $R_{\text {mesh }}$. Hence an escaped chain can diffuse many times its own length (past multiple potential binding sites) before rebinding. This result is consistent with a conceptual picture of a network linked together through well-formed coiled-coil junctions, in which most potential binding sites are fully occupied. Recently, Tang et al. invoked a non-zero bound state mobility in order to explain anomalous self-diffusion behavior observed by forced Rayleigh scattering (FRS) in a reversible protein hydrogel assembled from chains with four coiled-coil P blocks per chain. ${ }^{47}$ Bound mobility (possibly in the form of large clusters) is likely to be more significant in these gels, due to the much smaller fraction of free chains.

Tuning chain mobility with protein engineering. Reversible network association of the $\mathrm{P}$ domain reduces the effective diffusivity of PEP chains by two orders of magnitude. Given the programmability of coiled-coil assembly ${ }^{48}$ we imagined that it should be possible to control the effective diffusivity of a PEP chain by tuning the binding affinity of the P domain. In solution, coiled-coil assembly is driven by hydrophobic interactions between P domains. ${ }^{43}$ In the pentameric bundle, $48 \%$ of the total solvent-accessible area arising from the five individual helices is buried, demonstrating the critical role played by hydrophobic 
interactions in stabilizing the pentamer. ${ }^{27}$ We hypothesized that the hydrophobic leucine (Leu) contacts known to direct oligomerization of the $\mathrm{P}$ domain are also critical for reversible network association of a PEP chain.

Site-directed mutagenesis was performed on both ends of the original $\mathrm{PE}_{\mathrm{C}} \mathrm{P}$ probe to examine whether replacement of critical Leu residues would increase chain mobility. Guided by previous mutagenesis studies on the $\mathrm{P}$ domain, ${ }^{43}$ we made a single Leu $\rightarrow$ Ala mutation (L37A) within each terminal coil, which we predicted would reduce the thermodynamic driving force for oligomerization of the probe. L37 occupies the $a$-position of one of the heptad repeats of P (Figure 5A). Residues at the $a$ positions line the hydrophobic interior of the pentameric helical bundle, and their mutation to Ala destabilizes the assembly. ${ }^{43}$ We observed more rapid fluorescence recovery in PEP networks labeled with $\mathrm{PE}_{\mathrm{C}} \mathrm{P}-\mathrm{L} 37 \mathrm{~A}$ as compared to unmodified $\mathrm{PE}_{\mathrm{C}} \mathrm{P}$ (Figure 5A). We attribute the faster fluorescence recovery to a reduction in the strength of association of the mutant probe with network junctions, consistent with the previously reported low helicity and monomeric oligomerization state of $\mathrm{P}$ domains carrying the L37A mutation. ${ }^{43}$

The enhanced mobility of the $\mathrm{PE}_{\mathrm{C}} \mathrm{P}-\mathrm{L} 37 \mathrm{~A}$ probe illustrates the importance of hydrophobic interactions in network assembly, and suggests that increasing the hydrophobic character of the $\mathrm{P}$ domain should reduce chain mobility by increasing the strength of network association. We previously reported that replacement of Leu by $(2 S, 4 S)$-2-amino-4-methylhexanoic acid (homoisoleucine, Hil), a leucine surrogate with expanded hydrophobic surface area, significantly increases the thermostability of dimeric coiled-coil assemblies. ${ }^{49} \mathrm{We}$ hypothesized that replacement of the Leu residues in $\mathrm{PE}_{\mathrm{C}} \mathrm{P}$ by Hil (Figure $5 \mathrm{~A}$ ) might reduce probe mobility.

To test this hypothesis, we prepared $\mathrm{PE}_{\mathrm{C}} \mathrm{P}-\mathrm{Hil}$ probes in which ca. $92 \%$ of all Leu residues were replaced by Hil (see Supporting Information, Figures S8, S9 and Table S2 for details). In contrast to the accelerated recovery behavior of the $\mathrm{PE}_{\mathrm{C}} \mathrm{P}-\mathrm{L} 37 \mathrm{~A}$ mutant probe, recovery of the $\mathrm{PE}_{\mathrm{C}} \mathrm{P}-\mathrm{Hil}$ probe was slower than that of $\mathrm{PE}_{\mathrm{C}} \mathrm{P}$ (Figure 5B). Moreover, probes containing both Hil and Leu exhibited intermediate rates of recovery (ca. 53\% replacement, Figure S10). This confirms that the reduced rate of fluorescence recovery derives from a differential association of the $\mathrm{PE}_{\mathrm{C}} \mathrm{P}-\mathrm{Hil}$ probes with the PEP network junctions.

\section{CONCLUSION}

We have reported a FRAP-based method for characterizing strand exchange and polymer self-diffusivity in associative protein hydrogels. The application of this method relies on a novel 3 -state reaction-diffusion model of the strand exchange process. In this model, polymer chains move by a process called "hopping": the chains are free to diffuse spatially throughout the polymer network, unless trapped by reversible association with network junctions. This model fits our experimental FRAP curves well, and permits extraction of diffusion coefficients and equilibrium constants. We find that reversible network association exerts significant control over the effective mobility of individual chains. This allows the effective mobility " $D_{e f f}$ " to be tuned over a 500-fold range for probes that are all nominally the same size (Table 1), via simple changes in chain sequence. The formalism of the 3-state model also enables explicit prediction of $D_{\text {eff }}$ from an underlying knowledge of the binding strength $k_{o n}{ }^{*} / k_{\text {off }}$ and the free diffusivity $D_{f}$. The hopping mobility predicted by this formalism significantly underestimates the observed mobility. We interpret this discrepancy as indicating inequality of the equilibrium constants that control sequential binding to the network. Brownian Dynamics simulations support this interpretation, and suggest that the asymmetry in binding arises from an entropic constraint on the association of dangling chains due to local network structure. Importantly, such binding asymmetry is likely to be a general feature of reversible gels. Taken together, our results demonstrate that FRAP is wellsuited to probing diffusion and binding in protein hydrogels, and that facile protein engineering techniques afford a remarkable level of control over chain mobility in these systems.

Table 1. Summary of FRAP results calculated and predicted from recovery curves of engineered probes in $10 \%$ PEP hydrogels. Values represent mean \pm standard deviation $(a=10-12.5 \mu \mathrm{m}, n \geq 4$ recovery curves from at least 2 gel preparations per probe). Results for the PEC probe are determined from the 2-state model (eq. 3, $N=1$ ); $k_{o n}{ }^{*} / k_{o f f}$ for this probe reflects $K_{1}$. Results for PECP-type probes are calculated from the 3-state model (eq. 3, $N=2$ ) with $D_{f}=2.9 \pm 0.4 \times 10^{-8} \mathrm{~cm}^{2} \mathrm{~s}^{-1}$, and assuming $K_{1}=K_{2}=k_{o n}{ }^{*} / k_{o f f}$.

\begin{tabular}{|c|c|c|c|c|c|c|c|}
\hline Probe & $M w(k D a)$ & $D_{f}, D_{e f f}\left(10^{-10} \mathrm{~cm}^{2} \mathrm{~s}^{-1}\right)$ & $\boldsymbol{k}_{\text {on }}{ }^{*} / \boldsymbol{k}_{\text {off }}$ & {$[f]_{e q}$} & {$[b]_{e q}$} & $K_{d}(\boldsymbol{\mu M})$ & $\Delta G_{a}\left(\mathrm{kcal} \mathrm{mol}^{-11}\right)$ \\
\hline $\mathrm{AE}_{\mathrm{C}} \mathrm{A}$ & 20.9 & $270 \pm 190$ & - & 1.000 & - & - & - \\
\hline $\mathrm{E}_{\mathrm{C}}$ & 17.7 & $420 \pm 50$ & - & 1.000 & - & - & - \\
\hline $\mathrm{PEC}_{\mathrm{C}}$ & 25.4 & $12.3 \pm 1.4$ & $26.5 \pm 4.5$ & 0.036 & - & $47 \pm 5$ & $-5.9 \pm 0.1$ \\
\hline $\mathrm{PE}_{\mathrm{C}} \mathrm{P}$ pred & 32.2 & $0.4 \pm 0.1$ & $26.5 \pm 4.5$ & - & - & - & - \\
\hline $\mathrm{PE}_{\mathrm{C}} \mathrm{P} o b s$ & 32.2 & $2.1 \pm 0.5$ & $11.7 \pm 1.8$ & 0.007 & 0.914 & $108 \pm 13$ & $-5.4 \pm 0.1$ \\
\hline PECP-L37A & 32.1 & $51 \pm 17$ & $1.9 \pm 0.7$ & 0.174 & 0.531 & $720 \pm 190$ & $-4.3 \pm 0.2$ \\
\hline $\mathrm{PE}_{\mathrm{C}} \mathrm{P}-\mathrm{Hil}$ & 32.4 & $0.68 \pm 0.09$ & $20.3 \pm 1.4$ & 0.002 & 0.951 & $62 \pm 4$ & $-5.7 \pm 0.04$ \\
\hline
\end{tabular}

\section{EXPERIMENTAL}

Hydrogel Preparation. All protein concentrations are reported in $\%(w / v)$. To prepare a $10 \%(w / v)$ gel, $100 \mu \mathrm{L}$ of phosphate buffer (100 mM, pH 7.2 - 7.4) was added directly to 10 $\mathrm{mg}$ of lyophilized PEP and the suspension was placed on ice to promote gelation. After $2-4 \mathrm{~h}$ on ice, hydration was usually complete as evidenced by the formation of an optically clear gel. In order to ensure network homogeneity, gels were typically heated above the gel-sol transition temperature $\left(\sim 75^{\circ} \mathrm{C}\right.$ for a $10 \%$ gel) by submerging them in boiling water for $30-60$ s. Upon heating, even concentrated solutions of PEP (up to $30 \%$ ) became viscous liquids. After heating, samples were immediately placed back on ice to allow gels to reform. Alternatively, samples could be left on ice for $24-48 \mathrm{~h}$ without heating in order to obtain completely homogenous gels. Fluorescent hydrogels were prepared by adding low concentrations (typically 
mass ratios of 1:50 or 1:100 were used) of fluorescein-labeled probe chains to PEP networks.

Fluorescence Recovery after Photobleaching. Fluorescent hydrogels were placed between two glass slides separated by $120 \mu \mathrm{m}$ spacers (Secure-Seal spacer, $9 \mathrm{~mm} \times 0.12 \mathrm{~mm}$, Life Technologies). Photobleaching experiments were performed on a Zeiss LSM 5 Exciter inverted confocal microscope equipped with the following laser lines: 458, 488, 514, 543 and $633 \mathrm{~nm}$. All lasers were typically applied during the bleaching period. Cylindrical bleach volumes of defined radius were created using the bleach applet in the Zen 2009 confocal microscopy software suite (Zeiss). A 20X objective was used for the large spot size experiments $(a=12.5-25 \mu \mathrm{m}) .2000$ iterations at a scan rate of $1.61 \mu \mathrm{s}$ per pixel resulted in a well resolved cylindrical bleach volume that penetrated the entire gel. Fluorescence recovery in the photobleached spot was monitored between 500 and $530 \mathrm{~nm}$ with a wide pinhole on a single z-slice in the center of the hydrogel. Images were typically collected at a rate of $1 \mathrm{~s}^{-}$ ${ }^{1}$ and at a resolution of $256 \times 256$ pixels. Fluorescence intensities within the photobleached spot were quantified using the Zen region-of-interest "mean ROI" applet. To account for nonspecific photobleaching caused by image acquisition during spot recovery, all curves were normalized to the fluorescence intensity of a region far from the photobleached spot. Quantitative analysis of the fluorescence recovery curves was performed in MATLAB.

\section{ASSOCIATED CONTENT}

\section{Supporting Information}

Contains additional experimental details, mathematical derivations, and other supplementary information. This material is available free of charge via the Internet at http://pubs.acs.org.

\section{AUTHOR INFORMATION}

\section{Corresponding Author}

tirrell@caltech.edu

\section{Present Addresses}

*Chemistry Department, Smith College, Ford Hall, 100 Green Street, Northampton, MA 01063

\section{Funding Sources}

This work was supported by grant number DMR-1506483 from the Biomaterials Program of the U. S. National Science Foundation. A.K.O. acknowledges support by the National Science Foundation Graduate Research Fellowship under Grant No. DGE-1144469 and an HHMI Gilliam Fellowship.

\section{ACKNOWLEDGMENT}

We thank Steven Olsen and the Division of Chemistry and Chemical Engineering Instrument Shop for machining sample holders for the FRAP experiments. We also thank David Koos, Andres Collazo and the Biological Imaging Facility of the Caltech Beckman Institute for training and assistance in operating the confocal microscope. We thank John Bagert, Lawrence Dooling, and Cole DeForest for helpful discussions during the preparation of the manuscript.

\section{REFERENCES}

1. Banta, S.; Wheeldon, I. R.; Blenner, M. Аппи Rev Biomed Eng 2010, 12, 167-186.

2. Petka, W. A.; Harden, J. L.; McGrath, K. P.; Wirtz, D.; Tirrell, D. A. Science 1998, 281 (5375), 389-392.

3. Creutz, S.; van Stam, J.; De Schryver, F. C.; Jerome, R. Macromolecules 1998, 31 (3), 681-689.
4. Li, Z. L.; Dormidontova, E. E. Soft Matter 2011, 7 (9), 4179-4188.

5. Annable, T.; Buscall, R.; Ettelaie, R.; Whittlestone, D. J Rheol 1993, 37 (4), 695-726.

6. van der Heijden, T.; Modesti, M.; Hage, S.; Kanaar, R.; Wyman, C.; Dekker, C. Mol Cell 2008, 30 (4), 530-538.

7. Holthausen, J. T.; Wyman, C.; Kanaar, R. DNA Repair 2010, 9 (12), 1264-1272.

8. Shen, W.; Zhang, K. C.; Kornfield, J. A.; Tirrell, D. A. Nat. Mater. 2006, 5 (2), 153-158.

9. Shen, W.; Kornfield, J. A.; Tirrell, D. A. Macromolecules 2007, 40 (3), 689-692.

10. O'Shea, E. K.; Rutkowski, R.; Stafford, W. F.; Kim, P. S. Science 1989, 245 (4918), 646-648.

11. O'Shea, E. K.; Rutkowski, R.; Kim, P. S. Science 1989, 243 (4890), 538-542.

12. Landschulz, W. H.; Johnson, P. F.; Mcknight, S. L. Science 1988, 240 (4860), 1759-1764.

13. Olsen, B. D.; Kornfield, J. A.; Tirrell, D. A. Macromolecules 2010, 43 (21), 9094-9099.

14. Ryan, S. J.; Kennan, A. J. J. Am. Chem. Soc. 2007, 129 (33), 10255-10260.

15. Steinkruger, J. D.; Woolfson, D. N.; Gellman, S. H. J. Am. Chem. Soc. 2010, 132 (22), 7586-+.

16. Wendt, H.; Berger, C.; Baici, A.; Thomas, R. M.; Bosshard, H. R. Biochemistry 1995, 34 (12), 4097-4107.

17. Daugherty, D. L.; Gellman, S. H. J. Am. Chem. Soc. 1999, 121 (18), 4325-4333.

18. Sprague, B. L.; Pego, R. L.; Stavreva, D. A.; McNally, J. G. Biophys J 2004, 86 (6), 3473-3495.

19. Mayr, B. M.; Guzman, E.; Montminy, M. J Biol Chem 2005, 280 (15), 15103-15110.

20. McNally, J. G. Method Cell Biol 2008, 85, 329351.

21. Li, Y. A.; Kleijn, J. M.; Stuart, M. A. C.; Slaghek, T.; Timmermans, J.; Norde, W. Soft Matter 2011, 7 (5), 1926-1935.

22. DeSmedt, S. C.; Meyvis, T. K. L.; Demeester, J.; VanOostveldt, P.; Blonk, J. C. G.; Hennink, W. E. Macromolecules 1997, 30 (17), 4863-4870.

23. Branco, M. C.; Pochan, D. J.; Wagner, N. J.; Schneider, J. P. Biomaterials 2009, 30 (7), 1339-1347.

24. Herbst, F.; Seiffert, S.; Binder, W. H. Polym Chem 2012, 3 (11), 3084-3092.

25. Gribbon, P.; Hardingham, T. E. Biophys J 1998, 75 (2), 1032-1039.

26. Gribbon, P.; Heng, B. C.; Hardingham, T. E. Biophys J 1999, 77 (4), 2210-2216.

27. Malashkevich, V. N.; Kammerer, R. A.; Efimov, V. P.; Schulthess, T.; Engel, J. Science 1996, 274 (5288), 761765.

28. Perry, P. A.; Fitzgerald, M. A.; Gilbert, R. G. Biomacromolecules 2006, 7 (2), 521-530.

29. Tang, S.; Glassman, M. J.; Li, S.; Socrate, S.; Olsen, B. D. Macromolecules 2014, 47 (2), 791-799.

30. Soumpasis, D. M. Biophys J 1983, 41 (1), 95-97.

31. Hollenbeck, K. J., 1998. INVLAP.M: A MATLAB function for numerical inversion of Laplace transforms by the de Hoog algorithm.

http://www.mathworks.com/matlabcentral/answers/uploa ded files/1034/invlap.m. 
32. Zhang, W. B.; Sun, F.; Tirrell, D. A.; Arnold, F. H. J. Am. Chem. Soc. 2013, 135 (37), 13988-13997.

33. Zakeri, B.; Fierer, J. O.; Celik, E.; Chittock, E. C.; Schwarz-Linek, U.; Moy, V. T.; Howarth, M. Proc Natl Acad Sci USA 2012, 109 (12), E690-E697.

34. Cruise, G. M.; Scharp, D. S.; Hubbell, J. A. Biomaterials 1998, 19 (14), 1287-1294.

35. Weber, L. M.; Lopez, C. G.; Anseth, K. S. J Biomed Mater Res A 2009, 90 (3), 720-9.

36. Engberg, K.; Frank, C. W. Biomed Mater 2011, 6 (5), 055006.

37. Zitzewitz, J. A.; Bilsel, O.; Luo, J. B.; Jones, B. E.; Matthews, C. R. Biochemistry 1995, 34 (39), 12812-12819.

38. Patel, L. R.; Curran, T.; Kerppola, T. K. Proc Natl Acad Sci USA 1994, 91 (15), 7360-7364.

39. Ozeki, S.; Kato, T.; Holtzer, M. E.; Holtzer, A. Biopolymers 1991, 31 (8), 957-966.

40. Dalal, S.; Canet, D.; Kaiser, S. E.; Dobson, C. M.; Regan, L. Protein Eng Des Sel 2008, 21 (3), 197-206.

41. Park, S. Y.; Quezada, C. M.; Bilwes, A. M.; Crane, B. R. Biochemistry 2004, 43 (8), 2228-2240.
42. Wendt, H.; Baici, A.; Bosshard, H. R. J. Am. Chem. Soc. 1994, 116 (15), 6973-6974.

43. Gunasekar, S. K.; Asnani, M.; Limbad, C.; Haghpanah, J. S.; Hom, W.; Barra, H.; Nanda, S.; Lu, M.; Montclare, J. K. Biochemistry 2009, 48 (36), 8559-8567.

44. Beck, K.; Gambee, J. E.; Bohan, C. A.; Bachinger, H. P. J Mol Biol 1996, 256 (5), 909-923.

45. Kremer, K.; Grest, G. S. J Chem Phys 1990, 92 (8), 5057-5086.

46. Shen, W.; Kornfield, J. A.; Tirrell, D. A. Soft Matter 2007, 3 (1), 99-107.

47. Tang, S. C.; Wang, M. Z.; Olsen, B. D. J. Am. Chem. Soc. 2015, 137 (11), 3946-3957.

48. Harbury, P. B.; Zhang, T.; Kim, P. S.; Alber, T. Science 1993, 262 (5138), 1401-1407.

49. Van Deventer, J. A.; Fisk, J. D.; Tirrell, D. A. ChemBioChem 2011, 12 (5), 700-702. 


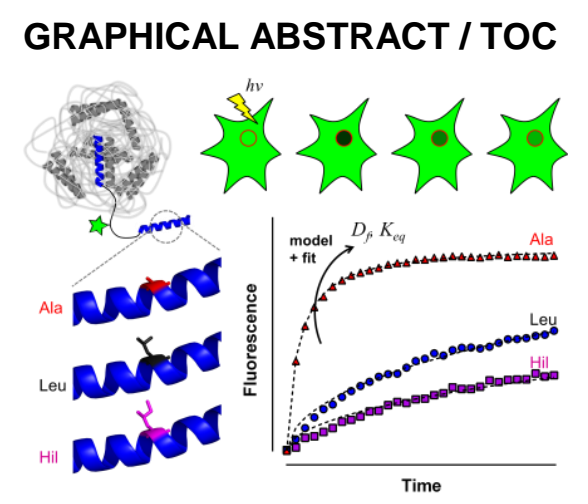

13

14

15

16

17

18

19

20

21

22

23

24

25

26

27

28

29

30

31

32

33

34

35

36

37

38

39

40

41

42

43

44

45

46

47

48

49

50

51

52

53

54

55

56

57

58

59

60 
A

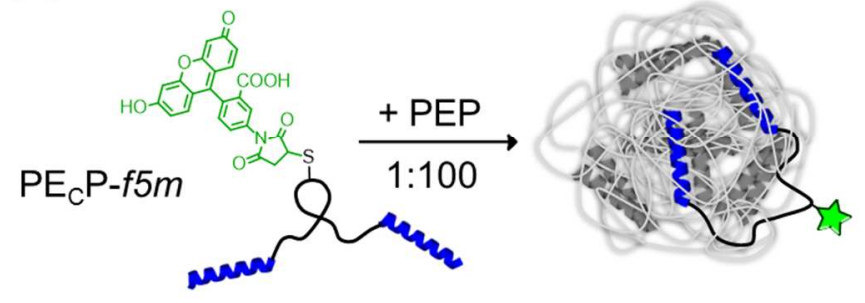

B
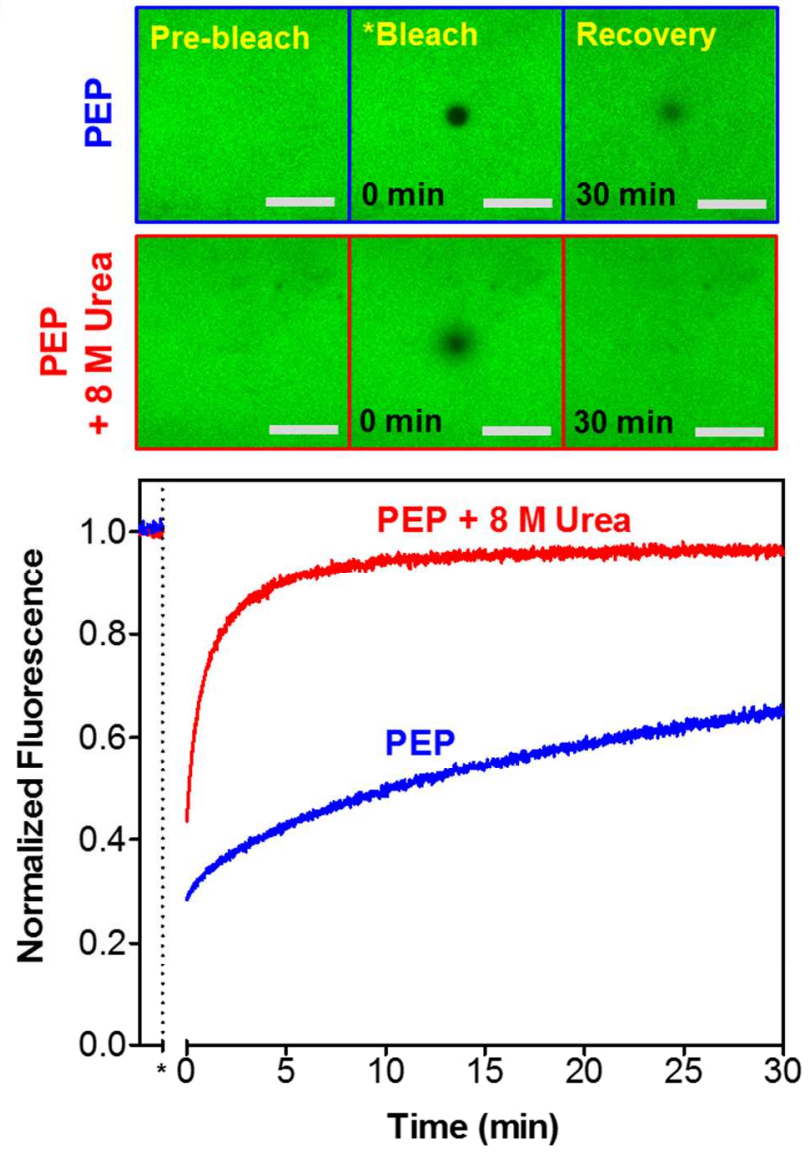

Figure 1

201×359mm (96 x 96 DPI) 

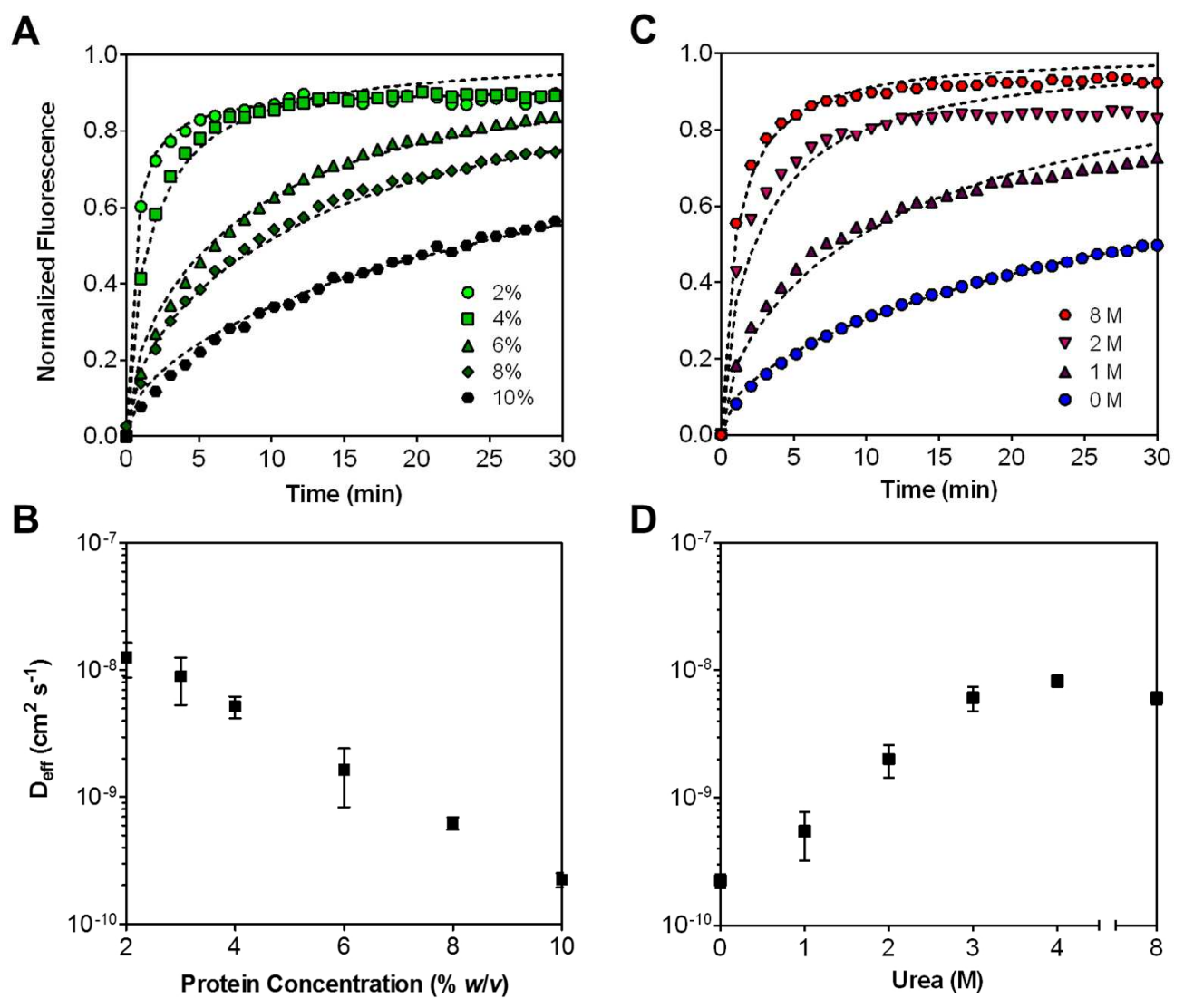

Figure 2

$411 \times 346 \mathrm{~mm}(96 \times 96 \mathrm{DPI})$ 
A

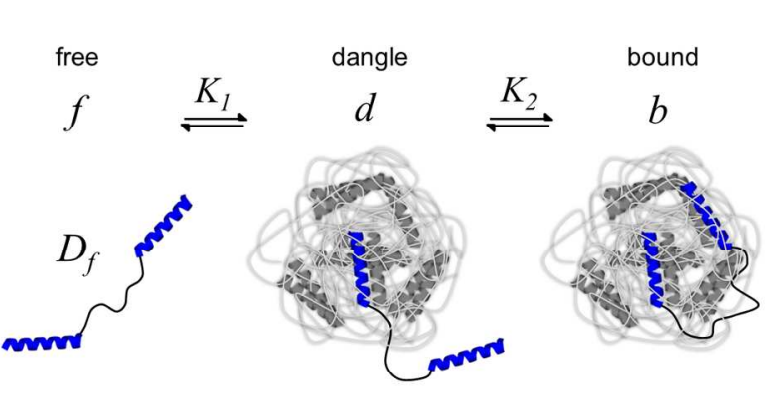

B
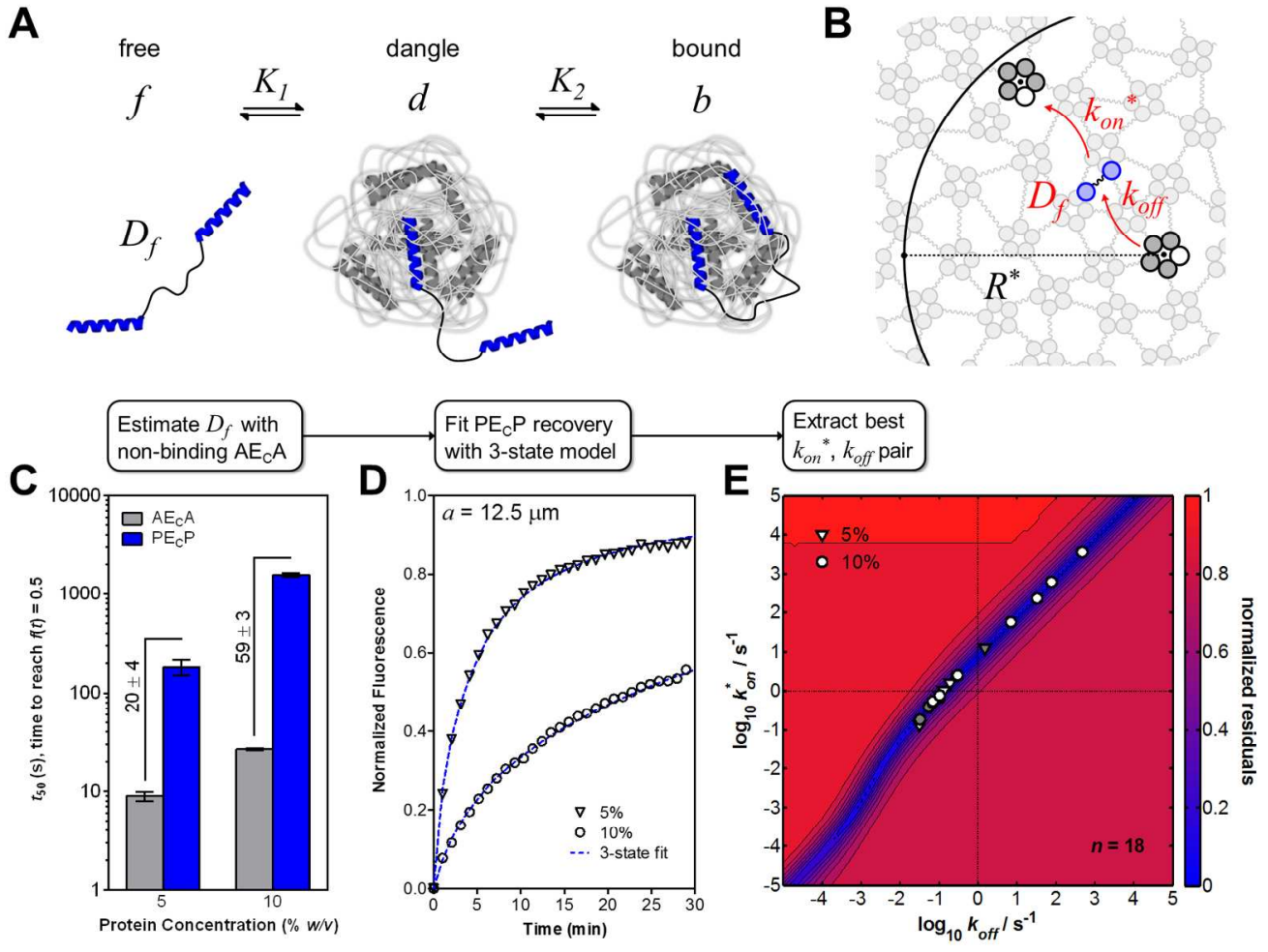

Figure 3

499×375mm (96 x 96 DPI) 
A

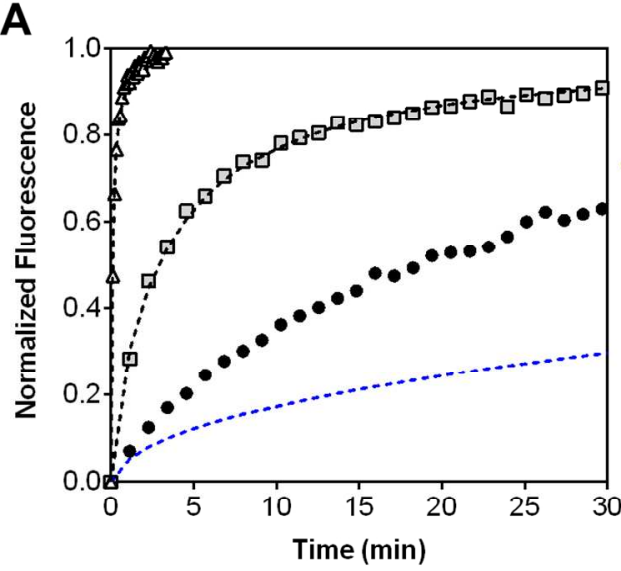

B

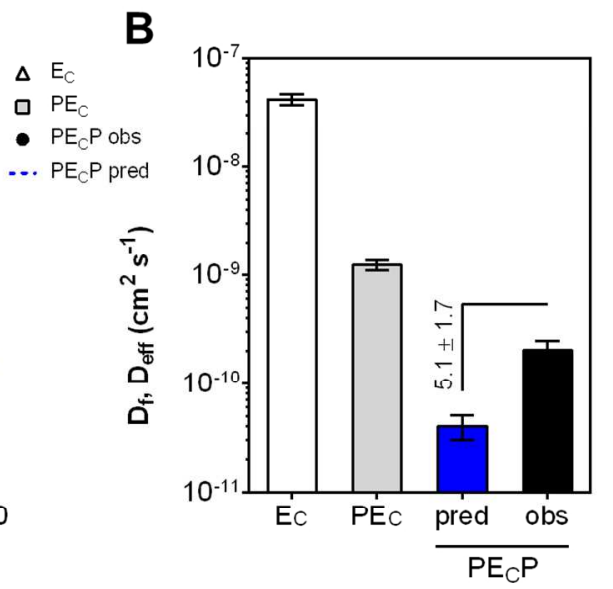

C

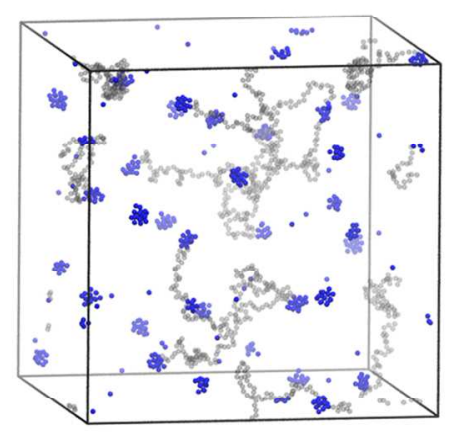

D

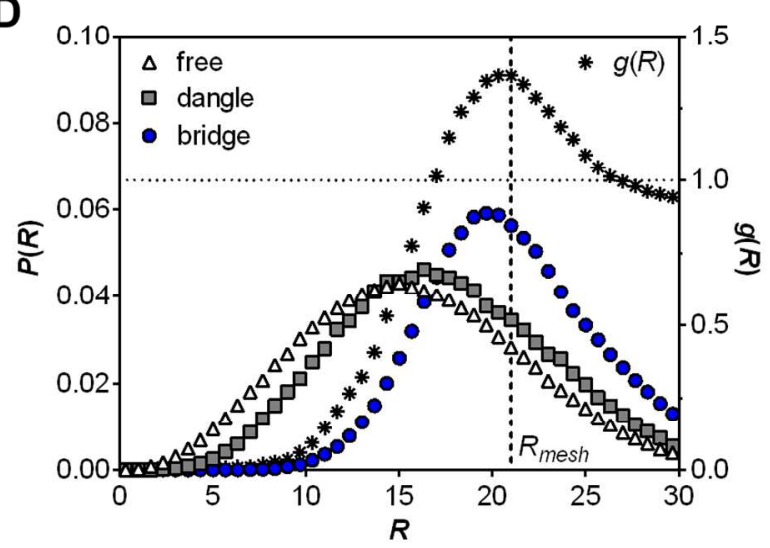

Figure 4

$377 \times 331 \mathrm{~mm}(96 \times 96 \mathrm{DPI})$ 
A
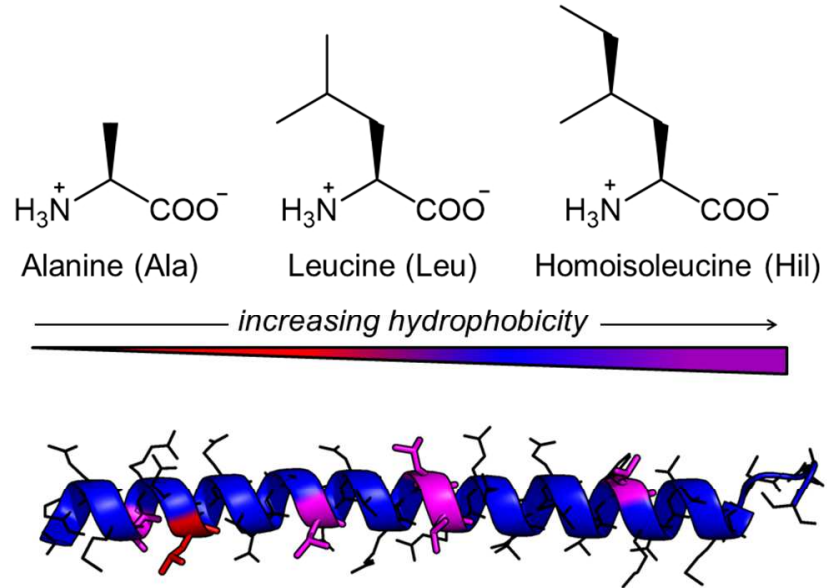

APQMLRE LQETNAA LQDVREL LRQQVKE ITFLKNT VMESDAS abcdefg |L37A

B

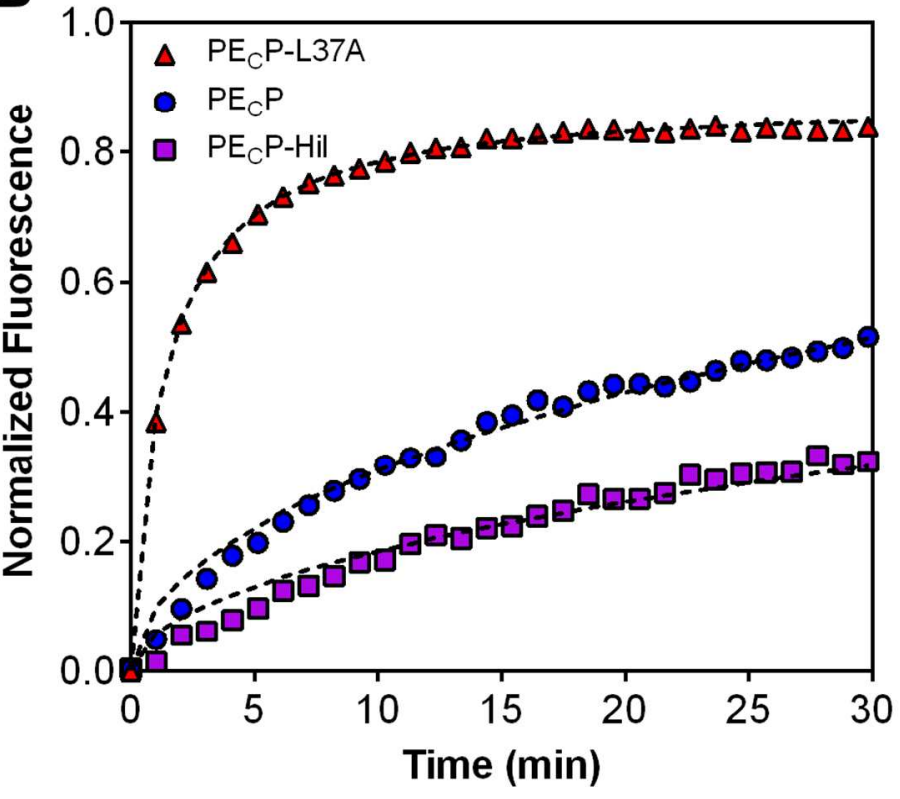

Figure 5

$260 \times 427 \mathrm{~mm}(96 \times 96 \mathrm{DPI})$ 


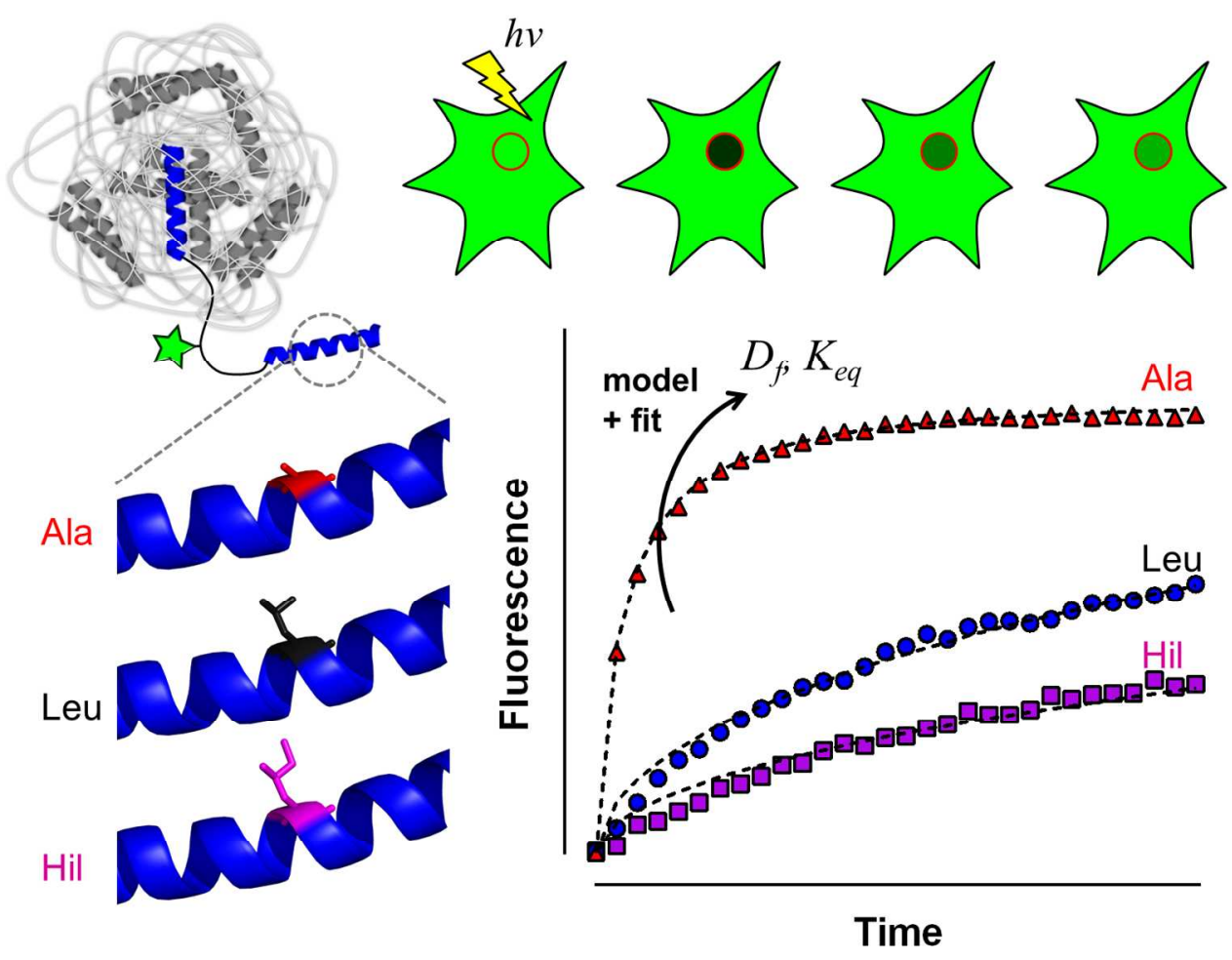

Table of Contents Graphic

$437 \times 330 \mathrm{~mm}(96 \times 96 \mathrm{DPI})$ 